\title{
Efeito da radiação gama e do armazenamento na oxidação lipídica e no colesterol de carne de cordeiros da raça Santa Inês
}

\author{
Effect of radiation and of storage in lipids oxidation
}

and cholesterol of lamb Santa Inês

\section{Adriana Régia Marques de SOUZA ${ }^{1}$, Valter ARTHUR ${ }^{1}$, Solange Guidolin CANNIATTI-BRAZACA ${ }^{2 *}$}

\begin{abstract}
Resumo
Vários fatores (peso, sexo, dieta e criação) influenciam na composição de ácidos graxos da carne de cordeiros. O presente estudo comparou os efeitos de três (3) doses de irradiação $(0,2,4 \mathrm{kGy})$ e do armazenamento refrigerado a $4{ }^{\circ} \mathrm{C}$ por 15 dias, nos valores de colesterol e TBARS (substâncias reativas ao ácido tiobarbitúrico) de carne de cordeiro tratados com diferentes dietas. Os valores de TBARS $(0,2722$ a 0,5186 mg.MDA. $\mathrm{kg}^{-1}$ ) aumentaram com o armazenamento e com a irradiação (3,5174 a 5,7202 mg.MDA.kg $\left.{ }^{-1}\right)$, indicando que ambos afetam a oxidação lipídica. As taxas de colesterol (56,64 a 79,29 mg. $\left.100 \mathrm{~g}^{-1}\right)$ diminuíram com o armazenamento $\left(32,53\right.$ a 53,54 mg.100 g $\left.{ }^{-1}\right)$, porém aumentaram com a dose de $2 \mathrm{kGy}$ para o segundo tempo de estocagem. A dieta também influenciou nos teores de TBARS e de colesterol da carne de cordeiros. A dieta controle foi a que apresentou valores de TBARS mais baixos, independentemente das doses de irradiação e do tempo de armazenamento. O colesterol se mostrou mais influenciável pelo tempo de armazenamento do que pelas doses de irradiação e dietas estudadas.

Palavras-chave: irradiação; TBA; colesterol; cordeiros, carne.
\end{abstract}

\begin{abstract}
Various factors (weigh, sex, diet and breeding) influence the composition of fatty acids from lamb. The present study compared the effects of the irradiation doses $(0,2,4 \mathrm{kGy})$ and refrigerated storage $\left(4^{\circ} \mathrm{C}\right)$ for 15 days in the cholesterol values and TBARS (substances reactivate to acid tiobarbituric) of lamb treated with different diets. The values of TBARS $\left(0.2722\right.$ to $0.5186 \mathrm{mg}$ MDA. $\left.\mathrm{kg}^{-1}\right)$ increased with storage and

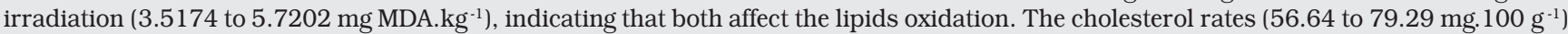
decreased with storage ( 32.53 to $53.54 \mathrm{mg} .100 \mathrm{~g}^{-1}$ ), however they increased with a dose of $2 \mathrm{kGy}$ for the second time of storage. The diet also influenced the levels of TBARS and of cholesterol from lamb. Diet control presented values independently from the irradiation doses lower than TBARS and of the time of storage. The cholesterol showed more influence for the time of storage than for the irradiation doses and studied diets.

Keywords: irradiation; TBA; cholesterol; lamb, meat.
\end{abstract}

\section{Introdução}

Entre os países desenvolvidos e em desenvolvimento, é crescente a demanda por produtos cárneos cozidos ou minimamente processados. Esses produtos são comercializados congelados, porém esta é uma técnica com custo elevado e instável quanto à segurança microbiológica. Além disso, o congelamento afeta a textura dos produtos e não elimina possíveis patógenos ${ }^{6}$. Uma alternativa é estocar estes produtos em estado refrigerado. Porém, a manutenção por refrigeração é válida para poucos dias, e a vida de prateleira dos produtos é um fator limitante da área de consumo. Uma tecnologia viável para estender a vida de prateleira e eliminar os patógenos de produtos refrigerados ou congelados é a irradiação.

A irradiação é o melhor tratamento para controle bacteriano em carnes frescas. Todavia, a radiação ionizante gera radicais livres que podem induzir a oxidação lipídica e outras mudanças químicas ${ }^{3}$. A oxidação lipídica é uma das maiores

\footnotetext{
Recebido para publicação em 21/2/2006

Aceito para publicação em 24/1/2007 (001672)

Laboratório de Irradiação de Alimentos e Radioentomologia,

Centro de Energia Nuclear na Agricultura - CENA,

Universidade de São Paulo - USP

Departamento de Agroindústria, Alimentos e Nutrição,

Escola Superior de Agricultura "Luiz de Queiroz" - ESALQ,

Universidade de São Paulo - USP,

Av. Pádua Dias, 11, CP 9, CEP 13418900, Piracicaba, SP - Brasil

E-mail:sgcbraza@esalq.usp.br

*A quem a correspondência deve ser enviada
}

causas de deterioração da qualidade da carne fresca ou cozida durante o armazenamento refrigerado ou congelado ${ }^{4}$.

Os lipídeos têm papel determinante na aceitação da carne, já que a sua concentração e composição influenciam fortemente as propriedades organolépticas (textura, sabor, cor e aroma). Os lipídeos apresentam composição similar em todas as espécies de animais, mesmo sob diferentes condições ambientais e de dieta $^{8}$. O fato de teores de lipídeos de origem animal ingeridos na dieta humana se apresentarem relacionados com doenças cardiovasculares, está promovendo a demanda por fontes de proteína animal que apresentem menores teores de lipídeos. Neste sentido, os cordeiros apresentam melhor qualidade de lipídeos intramuscular quando comparados com bovinos, suínos e frangos ${ }^{10}$.

A oxidação lipídica é o principal processo pelo qual ocorre perda de qualidade da carne e seus produtos, depois da deterioração microbiana. Além da alteração de odor e gosto, ela está relacionada também com a oxidação dos pigmentos da carne, provocando perda de cor. Alguns fatores afetam o processo de oxidação, entre eles, fatores ambientais (umidade, temperatura, luz e oxigênio), presença de metais (cobre, ferro e manganês), enzimas e pigmentos ${ }^{8}$.

A oxidação lipídica em carnes pode ser acompanhada por meio do valor de TBARS (substâncias reativas ao ácido 2-tiobarbitúrico) ${ }^{11}$, visto que produtos primários de oxidação 
lipídica constituem-se principalmente de hidroperóxidos, particularmente carbonilas, sendo o malonaldeído o elemento mais importante.

O colesterol é um composto relativamente estável, mas pode ser oxidado em certas condições. A oxidação do colesterol em carnes pode ser influenciada por alguns fatores como temperatura, tempo de estocagem, condições de embalagens e composição lipídica. A irradiação também pode ser um fator crucial para iniciar a oxidação do colesterol devido ao aumento da oxidação de ácidos graxos do sistema. Produtos da oxidação do colesterol (COPs) têm sido conhecidos por causar mais injúrias para as células arteriais que o colesterol puro e estão diretamente relacionados com o desenvolvimento de aterosclerose e doenças coronárias ${ }^{7}$.

Os objetivos desta pesquisa foram avaliar os efeitos do tempo de estocagem e da irradiação nos valores de colesterol e TBA de carne de cordeiros da raça Santa Inês que receberam diferentes dietas.

\section{Material e métodos}

\subsection{Material}

Cordeiros machos da raça Santa Inês foram divididos em três grupos com 4 animais por grupo, sendo o grupo 1 (controle) tratado com feno e concentrado (50-50\%). A suplementação da dieta para os outros dois grupos foi feita com taninos altamente concentrados (TAC) (extrato de Acácia molissima), sendo fornecidos na dose de $1,6 \mathrm{~g}^{\mathrm{kg}} \mathrm{kg}^{-1}$ de peso vivo. $\mathrm{O}$ grupo 2 (TAC1) foi suplementado com tanino por um dia, e o grupo 3 (TAC2) por dois dias, ambos nove dias antes do abate. Os cordeiros foram abatidos com 4 meses e após o abate foi separada a paleta (Infraspinatus, Triceps brachii, Supraspinatus) para realização das análises químicas.

As amostras da paleta de cordeiro da raça Santa Inês foram embaladas a vácuo 48 horas após o abate e armazenadas em geladeira $\left(4^{\circ} \mathrm{C}\right)$. Após a embalagem das paletas, estas foram levadas ao irradiador Multipropósito do Ipen (Instituto de Pesquisa Energéticas Nucleares), com fonte de ${ }^{60} \mathrm{Co}$ e a taxa de dose foi de $6 \mathrm{kGy}$ hora $^{-1}$. As amostras receberam doses de 2 e $4 \mathrm{kGy}$. As amostras não irradiadas serviram de controle. Enquanto as amostras foram irradiadas, as não irradiadas permaneceram em caixa de isopor com gelo, nas mesmas condições. Todas as amostras foram refrigeradas $\left(4^{\circ} \mathrm{C}\right)$.

As análises foram realizadas nas amostragem 1 (logo após a irradiação) e amostragem 2 (após 15 dias de armazenamento). A pesquisa foi realizada no Centro de Energia Nuclear na Agricultura (CENA) e na Escola Superior de Agricultura "Luiz de Queiroz" (ESALQ - USP).

\subsection{Análise colesterol}

Foi utilizada a metodologia de BLIGH e DYER ${ }^{1}$ para extração de lipídeos totais. Foram pesados $2 \mathrm{~g}$ de amostra, adicionaramse $10 \mathrm{~mL}$ de clorofórmio, $20 \mathrm{~mL}$ de metanol e $8 \mathrm{~mL}$ de água destilada, sendo agitados por 30 minutos. Foram adicionados, novamente, clorofórmio e sulfato de sódio $(1,5 \%)$ e agitados novamente. As camadas foram separadas, sendo desprezada a camada superior e filtrada a camada inferior. Para leitura do teor de colesterol, foi usado o método colorimétrico segundo BOHAC et al. ${ }^{2}$ : foram adicionados $10 \mathrm{~mL}$ de $\mathrm{KOH}$ ( $12 \%$ ) a $3 \mathrm{~mL}$ da amostra, sendo esta levada para banho-maria a $80^{\circ} \mathrm{C}$ por 15 minutos. Foram acrescentados $5 \mathrm{~mL}$ de água destilada à solução, que foi resfriada em torneira, e cloreto de ferro, e deixada em repouso por 15 minutos. Realizou-se a leitura da absorbância em 490 nm e os resultados foram expressos em $\mathrm{mg} .100 \mathrm{~g}^{-1}$.

\subsection{Análise de TBA}

As análises de TBA foram realizadas segundo metodologia descrita por KANATT, CHANDER e SHARMA ${ }^{6}$ : foram pesados 4 g de amostra, misturados a $16 \mathrm{~mL}$ de ácido tricloroacético (TCA) $5 \%$ e BHT (10 $\mu$ g.BHT.g ${ }^{-1}$ de lipídeo), sendo esta solução foi filtrada em papel de filtro. Foi acrescentado, ao filtrado, 0,02 M de TBA e aquecido em banho-maria a $45^{\circ} \mathrm{C}$ por 30 minutos. Depois de frio a leitura foi feita a $532 \mathrm{~nm}$. Os valores de TBARS foram expressos em mg de malonaldeído por $\mathrm{kg}$ de carne.

\subsection{Análise estatística}

O delineamento estatístico empregado foi o inteiramente casualizado, com três repetições por tratamento (amostra). Os resultados foram submetidos à análise de variância, com teste de F, e aos que apresentaram significância no nível de $1 \%$ foram realizados o teste de Tukey e as interações para estudar o efeito das doses de irradiação e do tempo de armazenamento na qualidade da carne. Os parâmetros estatísticos foram calculados pelo programa SAS (Statistical Analysis System) ${ }^{9}$.

\section{Resultados e discussão}

\subsection{Colesterol}

Os valores de colesterol encontrados para a paleta (Infraspinatus, Triceps brachii, Supraspinatus) de cordeiro Santa Inês estão expressos na Tabela 1 e nas Figuras 1 a 3.

Tabela 1. Valores de colesterol (mg. $100 \mathrm{~g}^{-1}$ ) encontrados para paleta de cordeiros Santa Inês tratados com diferentes dietas, doses de irradiação e tempo de armazenamento.

\begin{tabular}{ccccc}
\hline Dieta & $\begin{array}{c}\text { Tratamento } \\
\text { (kGy) }\end{array}$ & Dia 0 & Dia 15 & Prob $>$ F \\
\hline Controle & 0 & $79,29 \pm 0,67^{\mathrm{Ala} 2}$ & $38,53 \pm 0,19^{\mathrm{Ab}}$ & 0,01 \\
& 2 & $68,62 \pm 0,26^{\mathrm{ABa}}$ & $53,54 \pm 0,61^{\mathrm{Ba}}$ & 0,01 \\
& 4 & $58,43 \pm 1,19^{\mathrm{Ba}}$ & $41,12 \pm 0,33^{\mathrm{Cb}}$ & 0,01 \\
\hline TAC1 $^{*}$ & 0 & $76,21 \pm 0,79^{\mathrm{Aa}}$ & $32,53 \pm 0,22^{\mathrm{Ab}}$ & 0,01 \\
& 2 & $69,17 \pm 0,19^{\mathrm{Ba}}$ & $49,26 \pm 0,71^{\mathrm{Bb}}$ & 0,01 \\
& 4 & $66,59 \pm 0,45^{\mathrm{Ca}}$ & $45,85 \pm 0,90^{\mathrm{Cb}}$ & 0,01 \\
\hline TAC2 $^{*}$ & 0 & $75,92 \pm 0,39^{\mathrm{Aa}}$ & $30,48 \pm 0,65^{\mathrm{Ab}}$ & 0,01 \\
& 2 & $67,93 \pm 0,34^{\mathrm{Ba}}$ & $46,14 \pm 0,72^{\mathrm{Bb}}$ & 0,01 \\
& 4 & $62,25 \pm 0,69^{\mathrm{Ca}}$ & $40,31 \pm 0,38^{\mathrm{Cb}}$ & 0,01 \\
\hline
\end{tabular}

${ }^{1}$ Letras maiúsculas diferentes nas colunas para cada dieta indicam diferença significativa ( $1 \%)$ entre as doses; ${ }^{2}$ Letras minúsculas diferentes nas linhas indicam diferenças significativas (1\%) nos tempos de armazenamento; e *TAC1 e TAC2 - dietas com taninos altamente concentrados.

Somente para a dieta controle, no tempo 0, o tratamento que recebeu a dose de irradiação de 2 kGy não diferiu ( $p>0,01$ ) dos que receberam as demais doses. Para o tempo de 15 dias 
e as demais dietas, todas as doses foram significativamente diferentes, independentes do tempo de estocagem.

$\mathrm{O}$ armazenamento diminuiu significativamente as taxas de colesterol da carne de cordeiro independente das doses utilizadas. Isto pode ser explicado pela oxidação do colesterol em outros tipos de gorduras.

HOKE et al. ${ }^{5}$ encontraram valores de colesterol de $62,00 \mathrm{mg} .100 \mathrm{~g}^{-1}$ para paleta de cordeiros o que está de acordo com valores encontrados $\left(40,41\right.$ a $\left.79,29 \mathrm{mg} .100 \mathrm{~g}^{-1}\right)$ para as diferentes dietas.

Influência das dietas nos teores de colesterol - dose 0 kGy

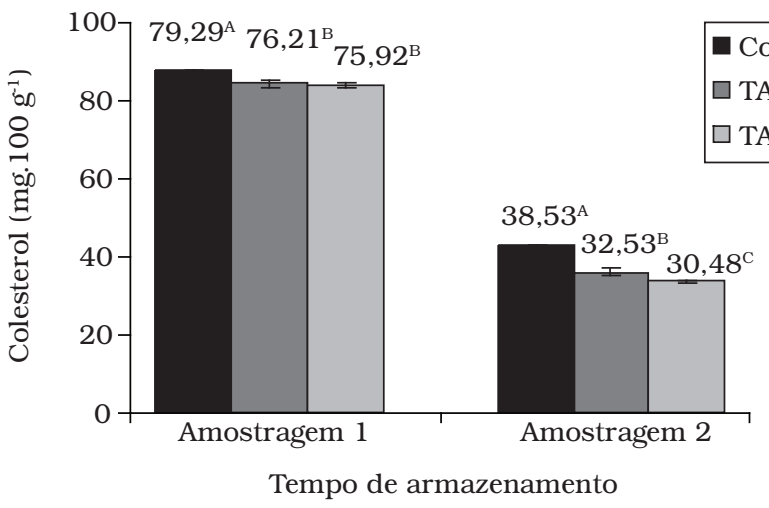

Figura 1. Influência das dietas e dos diferentes tempos de armazenamento nos teores de colesterol para dose $0 \mathrm{kGy}$.

Com a dose 0 kGy (Figura 1) houve tanto influência do tempo de armazenagem quanto das dietas nos valores de colesterol da carne. Para o tempo 0, as dietas suplementadas com taninos (TAC 1 e 2) não apresentaram diferença significativa ( $p>0,01$ ), sendo que a dieta controle foi a que teve mais afetados os níveis de colesterol. Porém, aos 15 dias de armazenagem, todas as dietas influenciaram nos teores de colesterol, sendo os valores mais baixos observados para a dieta TAC2 de 30,48 mg. $100 \mathrm{~g}^{-1}$.

Para a dose de 2 kGy, na amostragem 1 de armazenagem não houve diferença significativa entre as dietas, indicando que elas não influenciaram nos teores de colesterol. Na amostragem 2 ( 15 dias) de armazenagem, todas as dietas apresentaram diferenças significativas, indicando que, além das dietas, o tempo também contribui para os diferentes valores de colesterol encontrados na pesquisa (Figura 2).

As dietas influenciaram nos teores de colesterol, somente para a primeira amostragem para a dose de 4 kGy (Figura 3), na qual foi observado que todas as dietas foram significativamente diferentes ( $\mathrm{p} \leq 0,01)$. Para a segunda amostragem, tanto a carne dos cordeiros tratados com a dieta controle quanto a carne dos cordeiros tratados com a dieta TAC2 foram iguais.

\subsection{TBARS}

A oxidação lipídica foi medida em termos de substâncias reativas ao ácido tiobarbitúrico (TBARS) e os resultados são mostrados na Tabela 2 e nas Figuras de 4 a 6 .
Efeito das dietas nos teores de colesterol - dose $2 \mathrm{kGy}$

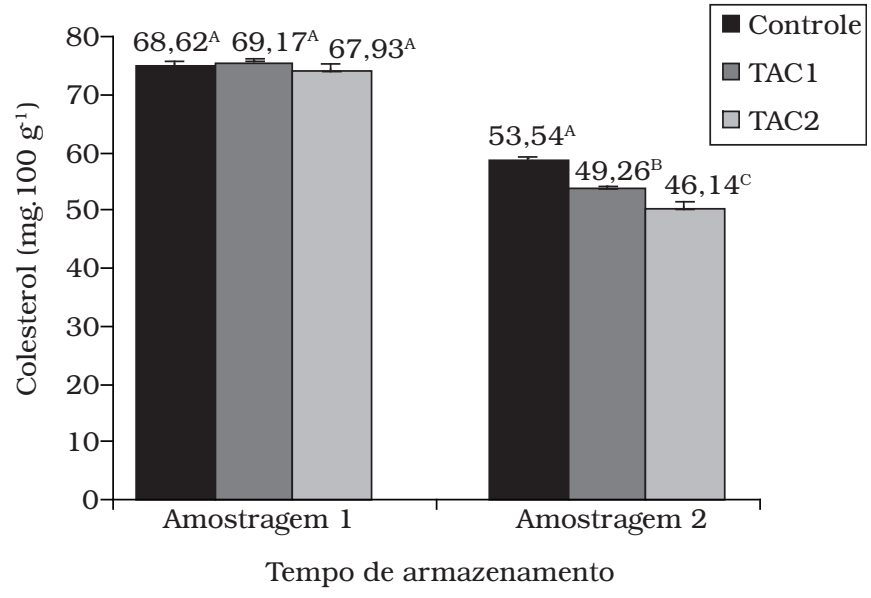

Figura 2. Influência das dietas e dos diferentes tempos de armazenamento nos teores de colesterol para dose $2 \mathrm{kGy}$.

Efeito das dietas nos teores de colesterol - dose 4 kGy

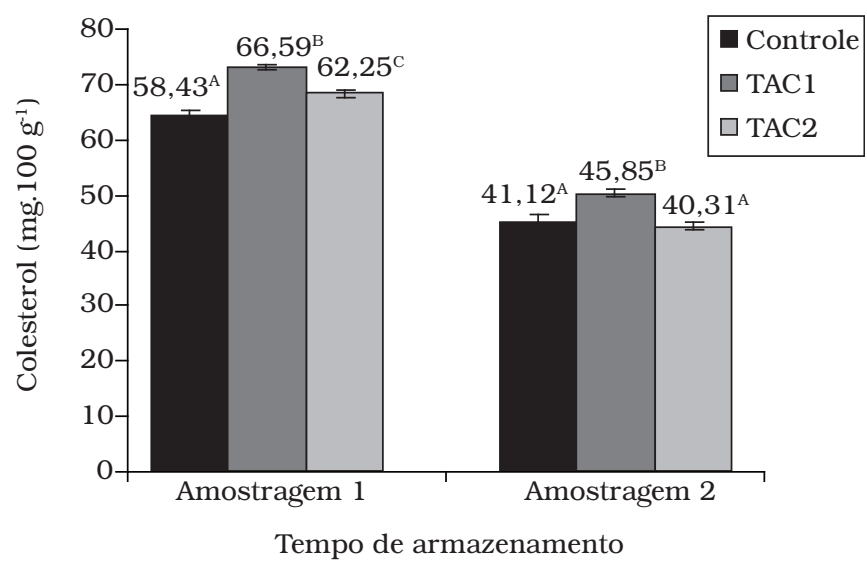

Figura 3. Influência das dietas e dos diferentes tempos de armazenamento nos teores de colesterol para dose $4 \mathrm{kGy}$.

A irradiação e o tempo de armazenamento influenciaram ( $\mathrm{p} \leq 0,01$ ) nos valores de TBARS da carne de cordeiro para todos os tratamentos. Depois de 15 dias de armazenamento, os valores de TBARS aumentaram significativamente $(\mathrm{p} \leq 0,01)$ para todas as amostras estudadas, irradiadas ou não. Independentemente da dieta dos cordeiros, os valores de TBARS foram afetados tanto pela radiação quanto pelo tempo de armazenamento. Os valores médios de TBARS das paletas variaram de 0,27 a 0,51, no início do experimento, e de 3,51 a $5,72 \mathrm{mg}$ de malonaldeído. $\mathrm{kg}^{-1}$, ao final dos 15 dias de armazenamento (Tabela 2), sendo o período de estocagem um fator preponderante para esta diferença. Este salto nos valores de TBARS provavelmente foi devido a um acúmulo generalizado de produtos secundários de ranço. Esse fato ocorreu para todos os tratamentos, visto que a dieta não influenciou os diferentes valores de TBARS. 
Para as dietas TAC1 e TAC2, somente houve diferença significativa ( $\mathrm{p} \leq 0,01)$ quanto ao efeito das doses no tempo 0 , nas amostras não irradiadas. Já para o tempo 15, todos os tratamentos demonstraram ser diferentes estatisticamente em cada dose.

No dia inicial, a dieta controle apresentou valores mais baixos, a dieta TAC1, valores intermediários, e a TAC2, os valores mais altos.

As dietas tiveram grande influência nos valores de TBARS encontrados para a amostragem 1 de armazenamento na dose de 0 kGy (Figura 4). Todas elas foram significativamente diferentes, mostrando que a dieta tem grande impacto na qualidade da carne de cordeiros. Mas o tempo também tem alta influência na qualidade da carne, como é visto na amostragem 2 de armazenamento, na qual, apesar do aumento nos valores de TBARS, as dietas foram estatisticamente iguais.

Tabela 2. Valores de TBARS (mg.MDA.kg ${ }^{-1}$ ) encontrados para paleta de cordeiros Santa Inês tratados com diferentes dietas, doses de irradiação e tempo de armazenamento.

\begin{tabular}{ccccc}
\hline Dieta & $\begin{array}{c}\text { Tratamento } \\
(\mathrm{kGy})\end{array}$ & Dia 0 & Dia 15 & Prob $>\mathrm{F}$ \\
\hline Controle & 0 & $0,2722 \pm 0,01^{\mathrm{Ala} 2}$ & $3,5174 \pm 0,01^{\mathrm{Ab}}$ & 0,01 \\
& 2 & $0,3054 \pm 0,01^{\mathrm{Ba}}$ & $4,5074 \pm 0,02^{\mathrm{Bb}}$ & 0,01 \\
& 4 & $0,3740 \pm 0,01^{\mathrm{Ca}}$ & $5,5505 \pm 0,02^{\mathrm{Cb}}$ & 0,01 \\
\hline TAC1 $^{*}$ & 0 & $0,3445 \pm 0,01^{\mathrm{Aa}}$ & $3,5395 \pm 0,02^{\mathrm{Ab}}$ & 0,01 \\
& 2 & $0,4065 \pm 0,01^{\mathrm{Ba}}$ & $4,6933 \pm 0,02^{\mathrm{Bb}}$ & 0,01 \\
& 4 & $0,4109 \pm 0,01^{\mathrm{Ba}}$ & $5,7047 \pm 0,01^{\mathrm{Cb}}$ & 0,01 \\
\hline TAC2 $^{*}$ & 0 & $0,4094 \pm 0,01^{\mathrm{Aa}}$ & $3,5572 \pm 0,03^{\mathrm{Ab}}$ & 0,01 \\
& 2 & $0,4125 \pm 0,01^{\mathrm{Aa}}$ & $4,7066 \pm 0,01^{\mathrm{Bb}}$ & 0,01 \\
& 4 & $0,5186 \pm 0,01^{\mathrm{Ba}}$ & $5,7202 \pm 0,02^{\mathrm{Cb}}$ & 0,01 \\
\hline
\end{tabular}

${ }^{1}$ Letras maiúsculas diferentes nas colunas para cada dieta indicam que há diferença significativa entre as doses: ${ }^{2}$ Letras minúsculas diferentes nas linhas indicam diferenças significativas nos tempos de armazenamento; and *TAC1 e TAC2 - dietas com taninos altamente concentrados.

Na dose de 2 kGy (Figura 5) as dietas TAC1 e TAC2 foram iguais em ambos os tempos de estocagem (amostragem 1 e 2). Mostrando que a dieta que mais influenciou nos valores de TBARS foi a dieta controle, na qual foram encontrados os menores valores.

Para dose de 4 kGy, todas as dietas apresentaram diferenças significativas na amostragem 1 . Já na amostragem 2, somente a dieta controle se mostrou diferente das demais dietas (Figura 6).

Nos valores de TBARS a dieta que teve maior alteração foi a controle, que apresentou valores mais baixos, independentemente do tempo de estocagem e das doses de irradiação estudadas.

\section{Conclusão}

Foram observadas diminuições nos teores de colesterol e aumento nos valores de TBARS, nas carnes de cordeiros da raça Santa Inês, em função das doses aplicadas e dos tempos de armazenamentos estudados.

A dieta controle foi a que manteve o valor mais baixo de TBARs.
Efeito das dietas nos teores de TBARS - dose $0 \mathrm{kGy}$

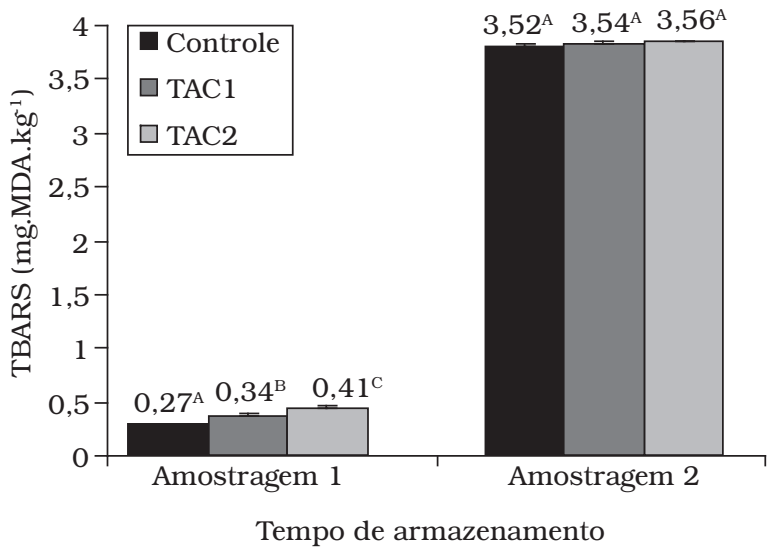

Figura 4. Influência das dietas e dos diferentes tempos de armazenamento nos valores de TBARS para dose 0 kGy.

Efeito das dietas nos teores de TBARS - dose $2 \mathrm{kGy}$

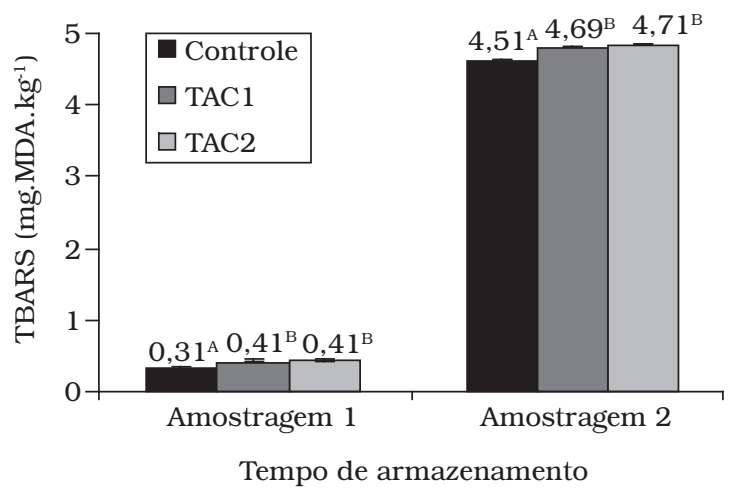

Figura 5. Influência das dietas e dos diferentes tempos de armazenamento nos valores de TBARS para dose $2 \mathrm{kGy}$.

Efeito das dietas nos teores de TBARS- dose $4 \mathrm{kGy}$

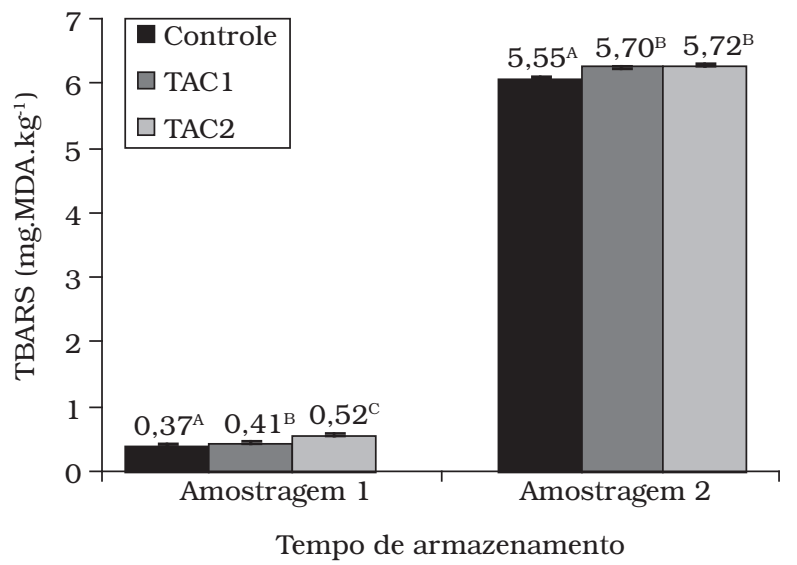

Figura 6. Influência das dietas e dos diferentes tempos de armazenamento nos valores de TBARS para dose 4 kGy. 


\section{Referências bibliográficas}

1. BLIGH, E. G.; DYER, W. J. A rapid method for total lipid extraction and purification. Canadian Journal of Biochemistry and Physiology, Ottawa, v. 37, n. 8, p. 911-917, aug. 1959.

2. BOHAC, C. E. et al. Assessment of methodologies for colorimetric cholesterol assay of meats. Journal of Food Science, Chicago, v. 53 , n. 6 , p. 1642-1644, nov. 1988.

3. DU, M. et al. Influence of dietary conjugated linoleic acid on volatile profiles, color and lipid oxidation of irradiated raw chicken meat. Meat Science, Illinois, v. 56, n. 4, p. 387-395, dez. 2000.

4. GOMES, H. A. et al. Evaluation of the 2-thiobarbituric acid method for the measurement of lipid oxidation in mechanically deboned gamma irradiated chicken meat. Food Chemistry, Oxford, v. 80, n. 3, p. 433-437, mar. 2003.

5. HOKE, I. M. et al. Nutrient and related food composition of exported Australian lambs cuts. Journal of Food Composition and Analysis, San Diego, v. 12, n. 2, p. 97-109, jun. 1999.

6. KANATT, S. R.; CHANDER, R.; SHARMA, A. Effect of radiation processing on the quality of chilled meat products. Meat Science, Oxford, v. 69, n. 2, p. 269-275, feb. 2005.
7. NAM, K. C. et al. Cholesterol oxidation products in irradiated raw meat with different packaging and storage time. Meat Science, Oxford, v. 58, n. 4, p. 431-435, aug. 2001.

8. PINO, L. M. Estabilidade oxidativa da carne de frangos alimentados com diferentes fontes lipídicas, armazenadas sob congelamento. 2005. 60 f. Dissertação (Mestrado em Ciência e Tecnologia de Alimentos), Escola Superior de Agricultura "Luiz de Queiroz", Universidade de São Paulo, Piracicaba, 2005.

9. SAS Institute. SAS user's guide: statistic. Version 6. 12 ed. Cary, SAS Institute, 2003, $846 \mathrm{p}$.

10. SOUZA, X. R. Efeitos de grupo genético, sexo e peso ao bate na qualidade de carne de cordeiros em crescimento. 2001. $119 \mathrm{f}$. Dissertação (Mestre em Ciência dos Alimentos), Universidade Federal de Lavras, Lavras, 2001.

11. TARLADGiS, B. G.; WATTS, B. M.; YOUNATHAN, M. T. A distillation method for the quantitative determination of malonaldehyde in rancid foods. Journal of the American Oil Chemists Society, Champaign, v. 37, n. 1, p. 44-48, jan. 1960. 